\title{
Traumatic midline subarachnoid hemorrhage on initial computed tomography as a marker of severe diffuse axonal injury
}

\author{
Daddy Mata-Mbemba, MD, PhD, ${ }^{1,2}$ Shunji Mugikura, MD, PhD, ${ }^{1}$ Atsuhiro Nakagawa, MD, PhD, ${ }^{2}$ \\ Takaki Murata, MD, PhD, ${ }^{1}$ Kiyoshi Ishii, MD, PhD, ${ }^{3}$ Shigeki Kushimoto, MD, PhD, ${ }^{4}$ \\ Teiji Tominaga, MD, PhD, ${ }^{2}$ Shoki Takahashi, MD, PhD, ${ }^{1}$ and Kei Takase, MD, PhD ${ }^{1}$ \\ Departments of ${ }^{1}$ Diagnostic Radiology, ${ }^{2}$ Neurosurgery, and ${ }^{4}$ Division of Emergency and Critical Care Medicine, Tohoku University
} Graduate School of Medicine; and 'Department of Radiology, Sendai Kousei Hospital, Sendai, Japan

\begin{abstract}
OBJECTIVE The objective of this study was to test the hypothesis that midline (interhemispheric or perimesencephalic) traumatic subarachnoid hemorrhage ( $\mathrm{SSAH}$ ) on initial CT may implicate the same shearing mechanism that underlies severe diffuse axonal injury (DAI).

METHODS The authors enrolled 270 consecutive patients (mean age [ \pm SD] $43 \pm 23.3$ years) with a history of head trauma who had undergone initial CT within 24 hours and brain MRI within 30 days. Six initial CT findings, including intraventricular hemorrhage (IVH) and tSAH, were used as candidate predictors of DAI. The presence of tSAH was determined at the cerebral convexities, sylvian fissures, sylvian vallecula, cerebellar folia, interhemispheric fissure, and perimesencephalic cisterns. Following MRI, patients were divided into negative and positive DAI groups, and were assigned to a DAI stage: 1) stage 0, negative DAl; 2) stage 1, DAl in lobar white matter or cerebellum; 3) stage 2, DAI involving the corpus callosum; and 4) stage 3, DAI involving the brainstem. Glasgow Outcome Scale-Extended (GOSE) scores were obtained in 232 patients.
\end{abstract}

RESULTS Of 270 patients, 77 (28.5\%) had DAl; tSAH and IVH were independently associated with DAI $(p<0.05)$. Of tSAH locations, midline tSAH was independently associated with both overall DAl and DAl stage 2 or 3 (severe DAl; $p<$ 0.05). The midline tSAH on initial CT had sensitivity of $60.8 \%$, specificity of $81.7 \%$, and positive and negative predictive values of $43.7 \%$ and $89.9 \%$, respectively, for severe DAI. When adjusted for admission Glasgow Coma Score, the midline tSAH independently predicted poor GOSE score at both hospital discharge and after 6 months.

CONCLUSIONS Midline tSAH could implicate the same shearing mechanism that underlies severe DAI, for which midline $\mathrm{TSAH}$ on initial CT is a probable surrogate.

https://thejns.org/doi/abs/10.3171/2017.6.JNS17466

KEY WORDS diffuse axonal injury; traumatic subarachnoid hemorrhage; traumatic brain injury; computed tomography; early outcome; long-term outcome; trauma

$\mathrm{T}$ RAUMATIC brain injury (TBI) constitutes the main cause of mortality and morbidity in young patients, and diffuse axonal injury (DAI) is thought to play a major role in these poor outcomes. ${ }^{15,24}$ Although the initial CT scan fails to accurately reveal DAI lesions, recent stud- ies have found that the presence of intraventricular hemorrhage (IVH) on the initial CT scan predicts the presence of DAI lesions in the corpus callosum or brainstem (severe DAI) ${ }^{18,20}$ which are the two DAI locations associated with poor clinical outcome. . $^{3,10,23}$

ABBREVIATIONS DAI = diffuse axonal injury; DWI = diffusion-weighted imaging; EDH = epidural hematoma; GCS = Glasgow Coma Scale; GOSE = Glasgow Outcome Scale-Extended; IVH = intraventricular hemorrhage; NPV = negative predictive value; PPV = positive predictive value; $\mathrm{SAH}=$ subarachnoid hemorrhage; SWI = susceptibility weighted imaging; TBI = traumatic brain injury; tSAH = traumatic SAH.

SUBMITTED February 23, 2017. ACCEPTED June 19, 2017.

INCLUDE WHEN CITING Published online January 5, 2018; DOI: 10.3171/2017.6.JNS17466. 
Evidence has shown that IVH as an isolated initial CT finding is extremely rare; it is almost always associated with subarachnoid hemorrhage (SAH) ${ }^{8,11,18}$ which is the most common posttraumatic intracranial lesion and seen in 33\%-60\% of patients. ${ }^{10}$ Furthermore, many studies have suggested an association between the presence of traumatic SAH (tSAH) on initial CT and poor clinical outcome. ${ }^{11-14}$ However, the mechanisms underlying the detrimental effect of tSAH are not yet understood, and an association between tSAH and DAI has never been established in previous studies.

Some authors have speculated that further exploration according to the location or severity of tSAH might help elucidate its clinical significance. ${ }^{12,13}$ Based on our clinical experience, we hypothesized that midline tSAH (interhemispheric or perimesencephalic), which is commonly associated with IVH on initial CT, may implicate the same shearing mechanism that underlies severe DAI. In the hope of clarifying the mechanism underlying poor clinical prognosis of some patients with tSAH, we retrospectively examined 270 consecutive patients who underwent brain MRI within 30 days after onset in our institution. We sought to examine: 1) the relationship between various initial CT findings, including tSAH, and the presence of DAI on MRI; and 2) the relationship between the locations of tSAH on initial CT and DAI staging on MRI, which has never been investigated to the best of our knowledge.

\section{Methods \\ Study Population}

This study had IRB approval with waiver of informed consent of patients in this retrospective interpretation of prospectively acquired data. Since January 1, 2009, we have prospectively maintained a database of patients with a reported history of TBI, facial injury, or both (head trauma), who were admitted to the emergency department of Tohoku University Hospital, a major tertiary referral hospital in northeastern Japan. Because TBI of all severities is so frequently accompanied by facial injury, ${ }^{4,27}$ and the degree of coexistence between these lesions sometimes cannot be accurately established on clinical grounds without imaging, we believed that it was appropriate to assess all of these patients together. Therefore, we have enrolled in our database 1189 consecutive patients with a reported history of closed head trauma who underwent a screening brain CT scan within 24 hours of injury between January 1, 2009, and December 31, 2013. Of the 1189 patients, we retrospectively identified 270 consecutive patients (age range 1-94 years, mean [ $\pm \mathrm{SD}] 43 \pm 23.3$ years) who underwent brain MRI within 30 days, following inclusion criteria in similar previous studies on DAI..$^{3,6}$

At the time of their admission to the emergency department, clinical symptoms of TBI were mild (Glasgow Coma Scale [GCS] score 13-15) in 194 patients (71.9\%), moderate (GCS score 9-12) in $41(15.2 \%)$, and severe (GCS score 8 or less) in 35 (12.9\%).

\section{CT Evaluation}

CT images were obtained as part of standard daily clinical care at our institution, using a 16-slice CT scan- ner (Bright Speed Elite 2006, GE Healthcare) located in the emergency room. Only initial nonenhanced CT scans obtained within 24 hours after sustaining the head injury were considered in this study. At our institution, in patients with unstable clinical conditions, resuscitation and hemodynamic stabilization are achieved prior to CT study.

Blinded to clinical information and the MRI findings of patients, a second-year neuroradiology fellow (D.M.M.) and an attending neuroradiologist (S.M.) with 4 and 15 years of experience, respectively, in the imaging of neurotrauma independently reviewed the initial CT scans. Consensus was used to solve disagreements between the 2 readers. The readers' data before consensus were used to calculate the agreement between the two readers.

Although the main purpose of this study was to document the clinical significance of tSAH and its locations in relation to severe DAI, we believed that it was relevant to first assess the clinical value of tSAH among other initial CT findings with respect to the overall DAI disease. Therefore, as candidate CT predictors of DAI, we chose to use the following 5 initial CT items whose clinical significance are sufficiently validated in Marshall and Rotterdam CT scores: ${ }^{14,16,17,18}$ basal cistern status, presence of midline shift, presence of epidural hematoma (EDH), volume of the hemorrhagic mass, and presence of IVH and/or tSAH. The 5 initial CT items were defined and measured according to previous reports..$^{14,16-18}$

Regarding tSAH, we specifically determined its presence or not at the following 6 locations: cerebral convexities, sylvian fissures, sylvian vallecula (i.e, CSF spaces between frontal and temporal lobes containing the horizontal branch of the middle cerebral artery), interhemispheric fissure, cerebellar folia, and perimesencephalic cisterns (including the interpeduncular fossa, ambient cisterns, and quadrigeminal cistern).

\section{Evaluation of MRI}

MRI studies in our patients were performed using one of the two 1.5-T MRI units (Signa Horizon LX, GE Medical Systems, or Intera Achieva Nova Dual, Philips Medical Systems) equipped with a conventional head coil. Patients were randomly allocated to either MRI machine for their scans.

Blinded to clinical information and CT findings of patients, two neuroradiologists (T.M. and S.T.) with 10 and 25 years of experience, respectively, independently reviewed the initial MRI for the presence of DAI using diffusionweighted imaging (DWI), axial FLAIR imaging, and axial turbo spin echo T2-weighted imaging for nonhemorrhagic DAI and axial $\mathrm{T} 2 *$-weighted gradient echo imaging and susceptibility weighted imaging (SWI, additionally performed in 17 patients) for hemorrhagic DAI. As grading of DAI on MRI was one of the outcomes in this study, to ensure appropriate assignment of patients to groups, each reader reviewed MR images twice with the second review occurring at least a month after and blind to the first review to minimize bias. Consensus was used to solve disagreements between reviewers. The reviewers' data before consensus were used to calculate interobserver and intraobserver agreement.

Hemorrhagic and nonhemorrhagic DAI were defined 
TABLE 1. Demographic and clinical characteristics of patients

\begin{tabular}{|c|c|c|c|c|}
\hline \multirow[b]{2}{*}{ Variable } & \multirow[b]{2}{*}{ No. } & \multicolumn{2}{|c|}{ DAI } & \multirow{2}{*}{$\begin{array}{c}p \\
\text { Value }\end{array}$} \\
\hline & & Present & Absent & \\
\hline No. of patients & 270 & $77(28.5)$ & $193(71.5)$ & \\
\hline Mean age \pm SD (yrs) & $43 \pm 23.3$ & $41.2 \pm 21.9$ & $43.8 \pm 23.9$ & 0.3870 \\
\hline Sex & & & & 0.8869 \\
\hline Male & $198(73.3)$ & $56(72.7)$ & $142(73.6)$ & \\
\hline Female & $72(26.7)$ & $21(27.3)$ & $51(26.4)$ & \\
\hline Mean GCS score \pm SD & $12.6 \pm 3.2$ & $10.7 \pm 3.8$ & $13.3 \pm 2.6$ & $<0.0001$ \\
\hline Cause of accident & & & & 0.1176 \\
\hline Traffic accident & $160(59.3)$ & $54(70.1)$ & $106(54.9)$ & \\
\hline Fall & $93(34.4)$ & $22(28.6)$ & $71(36.8)$ & \\
\hline Others & $17(6.3)$ & $1(1.3)$ & $16(8.3)$ & \\
\hline $\begin{array}{l}\text { Median time from onset until MRI in days ( } 25 \text { th, } 75 \text { th percen- } \\
\text { tile) }\end{array}$ & $4(1,9)$ & $4(2,8.5)$ & $3(1,10)$ & 0.3986 \\
\hline Median GOSE score at discharge (25th, 75th percentile) ${ }^{*}$ & $7(4,8)$ & $5(4,7)$ & $7(5,8)$ & $<0.0001$ \\
\hline Median GOSE score 6 mos later (25th, 75th percentile) ${ }^{*}$ & $8(5.25,8)$ & $7(4.5,8)$ & $8(6,8)$ & $<0.0001$ \\
\hline
\end{tabular}

according to previous studies. ${ }^{20,25}$ Based on the MRI results, we classified all 270 patients into two settings. First, patients were categorized as DAI (hemorrhagic and nonhemorrhagic) or no DAI. Then, patients were assigned to 1 of the 4 DAI stages, ${ }^{1,5}$ a measure that was reported to significantly predict outcome in patients. These 4 DAI stages are: 1) stage 0 , no DAI; 2) stage 1, DAI lesions confined to the lobar white matter or cerebellum; 3) stage 2, DAI lesions located in the corpus callosum with or without lesions of stage 1; and 4) stage 3 (the worst), DAI lesions located in the brainstem with or without lesions of stages 1 and/or 2 .

\section{Clinical Outcome}

Neurological impairment was assessed by the 8-point Glasgow Outcome Scale-Extended (GOSE) applied by a neurosurgeon (A.N.) with 10 years of experience in head trauma management, using a structured interview. ${ }^{6,26}$ Poor outcome was defined as a GOSE score $\leq 4$.

Of the 270 patients, $16(5.9 \%)$ were lost at the 6-month follow-up and 24 patients $(8.9 \%)$ less than 17 years old were excluded because the GOSE was developed for adult patients only. ${ }^{22,23}$ Finally, the GOSE score was obtained in 232 patients $(85.9 \%)$ at hospital discharge (early) and 6 months after injury (long term).

\section{Analysis and Statistics}

To test the hypothesis that the presence of tSAH on initial CT was associated with overall DAI, we examined the relationship between all 6 initial CT findings, including $\mathrm{tSAH}$ and IVH, and the presence of DAI on MRI using multivariate logistic regression. We ran the test twice, i.e., the variables tSAH and IVH were put into the logistic regression model first, combined into 1 variable (IVH/tSAH) as included in the Rotterdam score. ${ }^{14}$ Next, we separated IVH/tSAH into two different items of IVH and $\mathrm{tSAH} .{ }^{17}$ When $\mathrm{tSAH}$ was proved to be associated with DAI, we further examined the relationship between tSAH locations and both overall DAI and severe DAI.

Finally, when an association between midline tSAH on initial CT and severe DAI was found, we assessed the clinical relevance of midline SAH. First, we calculated the sensitivity, specificity, and positive and negative predictive values (PPVs and NPVs) of midline tSAH in the prediction of severe DAI. Next, midline tSAH and IVH were adjusted for GCS score in the prediction of poor outcome (GOSE) using multiple logistic regression.

All statistical analyses were performed using JMP Pro software (version 10, SAS Institute, Inc.) and p values < 0.05 were considered statistically significant.

\section{Results \\ DAI Detected by MRI}

The two neuroradiologists agreed on DAI stages in 254 of 270 patients (weighted kappa $=95.9 \%$ ). The intraobserver agreement was $98 \%$ for reader 1 and $99 \%$ for reader 2 .

Of the 270 patients, 77 (28.5\%) had DAI on MRI. Regarding the stage of DAI, 193 patients $(71.5 \%)$ had DAI stage 0 (no DAI), 26 patients $(9.6 \%)$ had stage 1,31 patients $(11.5 \%)$ has stage 2 , and 20 patients $(7.4 \%)$ had stage 3 . The majority $(74 \%, 57$ of 77 ) of patients with DAI showed coexistence of hemorrhagic and nonhemorrhagic DAI lesions. Hemorrhagic DAI lesions alone were noted in 12 patients $(15.6 \%)$, whereas nonhemorrhagic DAI lesions alone were detected in $8(10.4 \%)$.

\section{Patient Clinico-Demographics and DAI}

The GCS score was significantly lower in patients with DAI ( $\mathrm{p}<0.0001$, Table 1). No statistically significant differences were noted between the DAI and no-DAI groups 
TABLE 2. Relationship between the 6 initial CT findings and the presence of DAI on subsequent MRI using multivariate logistic regression

\begin{tabular}{lcccc}
\hline & & \multicolumn{3}{c}{ Multivariate Analysis } \\
\cline { 3 - 5 } CT Items & No. & Adjusted OR & $95 \% \mathrm{Cl}$ & $\mathrm{p}$ Value \\
\hline Basal cistern status & & & & \\
\hline Normal & $257(95)$ & 1.1 & $0-45.4$ & 0.9410 \\
\hline Compressed & $9(3)$ & 2 & $0.2-29.7$ & 0.5712 \\
\hline Absent & $4(1)$ & 2.4 & $0-133.9$ & 0.6524 \\
\hline Midline shift & $15(6)$ & 1.3 & $0-48.7$ & 0.8583 \\
\hline EDH & $19(7)$ & 1.4 & $0.4-4.4$ & 0.5405 \\
\hline tSAH & $114(43)$ & 3.6 & $2-7$ & $<0.0001$ \\
\hline IVH & $28(10)$ & 3 & $1.3-7.6$ & 0.0130 \\
\hline Volume of hemor- & & & & \\
rhagic mass (ml) & & & & \\
\hline$\quad$ Absent & $188(70)$ & 0.9 & $0.4-1.8$ & 0.8024 \\
\hline$<25$ & $68(25)$ & 0.5 & $0-2.2$ & 0.4946 \\
\hline$\quad 25$ & $14(5)$ & 0.5 & $0-2.2$ & 0.3769 \\
\hline
\end{tabular}

Data given as number of patients (\%) unless otherwise indicated. Boldface type indicates statistical significance.

regarding age, sex, cause of injury, or time between injury and initial MRI of patients ( $p>0.05$ ).

Of 232 patients assessed with the GOSE, poor outcomes were noted in 63 patients (27.1\%) at hospital discharge and in 38 patients (16.4\%) at 6 months after injury. The DAI stage was significantly and negatively correlated with GOSE score at discharge $(\rho=-0.30, p<0.0001)$ and at 6 months $(\rho=-0.29, p<0.0001)$. Patients with severe DAI (stage 2 or 3 ) showed significantly poor GOSE score at both discharge $(p=0.0003)$ and at 6 months $(p=$ 0.0001).

\section{Relationship Between Initial CT Findings and DAI}

In our study, overall tSAH was noted in 114 patients (43\%), among whom midline tSAH was found in 72. Of 77 patients with DAI, $52(67.5 \%)$ demonstrated tSAH with 38 of them having midline tSAH (49.4\%), while 14 patients $(18.2 \%)$ showed tSAH only in a location other than midline. Of 51 patients with severe DAI, 40 (78.4\%) dem- onstrated tSAH with 31 of them $(60.8 \%)$ having midline $\mathrm{SAH}$, while 9 patients (17.6\%) showed tSAH only in a location other than midline.

IVH was noted in 28 (10.4\%) of 270 patients (Table 2). Only $1(3.6 \%)$ of the 28 patients with IVH exhibited IVH as the singular CT finding, while 26 patients $(92.8 \%)$ had IVH associated with tSAH, and another patient (3.6\%) had IVH without tSAH but with a contusion on initial CT. Conversely, $22.8 \%$ of the 114 patients with tSAH had IVH and $29.1 \%$ of the 72 patients with midline tSAH had associated IVH.

In the setting of IVH combined with tSAH in 1 variable, of the 5 initial CT findings, the item of IVH/tSAH was independently associated with DAI (adjusted odds ratio $[\mathrm{OR}]=5.2, \mathrm{p}<0.0001)$. When $\mathrm{IVH} / \mathrm{tSAH}$ was split into two separate variables of IVH and tSAH, of the 6 initial CT findings, the items of IVH and tSAH were independently associated with DAI (IVH adjusted OR $=3.0$, $\mathrm{p}=0.0130 ;$ tSAH adjusted $\mathrm{OR}=3.6, \mathrm{p}<0.0001$; Table 2).

\section{Relationship Between the Location of tSAH and DAI}

Only the two midline (interhemispheric and perimesencephalic) tSAH locations were independently associated with overall DAI disease (both $\mathrm{p}<0.05$ ) and severe DAI (both $p<0.05$; Table 3, Figs. 1 and 2). Even when adjusted for other initial CT findings, including IVH, the two midline tSAH locations (both $\mathrm{p}<0.05$ ) remained independently associated with DAI, along with IVH (Table 4).

\section{Clinical Relevance of Midline SAH}

The presence of midline tSAH on initial CT had a sensitivity of $60.8 \%$, specificity of $81.7 \%$, PPV of $43.7 \%$, and NPV of $89.9 \%$ in predicting severe DAI. The admission GCS score and presence of midline SAH were independently associated with poor outcomes at both hospital discharge (GCS score OR $=18.1, \mathrm{p}<0.0001$; midline tSAH $\mathrm{OR}=2.7, \mathrm{p}<0.007)$ and 6 months after injury (GCS score $\mathrm{OR}=6.4, \mathrm{p}=0.0125$; midline $\mathrm{tSAH} \mathrm{OR}=2.7, \mathrm{p}=0.0157$ ).

\section{Discussion}

We have documented that tSAH and IVH detected on initial CT were independently associated with DAI on subsequent MRI. Of the tSAH locations, the midline (interhemispheric and perimesencephalic) tSAH specifically

TABLE 3. Results of multivariate logistic regressions of the 6 locations of tSAH on initial CT in predicting overall DAl or severe DAI disease

\begin{tabular}{|c|c|c|c|c|c|c|c|}
\hline \multirow[b]{2}{*}{ tSAH locations } & \multirow{2}{*}{$\begin{array}{l}\text { No. } \\
(\%)\end{array}$} & \multicolumn{3}{|c|}{ Overall DAI Disease (Present vs Absent)* } & \multicolumn{3}{|c|}{ Severe DAI (Present vs Absent)† } \\
\hline & & Adjusted OR & $95 \% \mathrm{Cl}$ & $p$ Value & Adjusted OR & $95 \% \mathrm{Cl}$ & p Value \\
\hline Cortex & $100(37)$ & 1.7 & $0.6-4.0$ & 0.2520 & 2.5 & $0.9-6.5$ & 0.0802 \\
\hline Sylvian fissure & $69(26)$ & 1.7 & $0.6-4.1$ & 0.2634 & 1.5 & $0.6-3.8$ & 0.4012 \\
\hline Sylvian vallecula & $50(19)$ & 1.3 & $0.5-3.4$ & 0.6109 & 1.1 & $0.4-2.8$ & 0.8258 \\
\hline Interhemispheric fissure & $27(10)$ & 5.8 & $1.9-21.4$ & 0.0015 & 3.4 & $1.1-12.1$ & 0.0322 \\
\hline Cerebellar folia & $13(5)$ & 2 & $0.5-8.2$ & 0.3319 & 2.0 & $0.5-7.9$ & 0.3432 \\
\hline Perimesencephalic cisterns & $57(21)$ & 5.4 & $2.4-13.1$ & $<0.0001$ & 4.2 & $1.8-10.2$ & 0.0007 \\
\hline
\end{tabular}

Boldface type indicates statistical significance.

* Overall DAI disease $=$ DAl of any stage $(1,2$, or 3$)$, present or not.

$\dagger$ Severe DAI = presence of DAl located in the corpus callosum, brainstem, or both (DAl stage 2 or 3 ). 

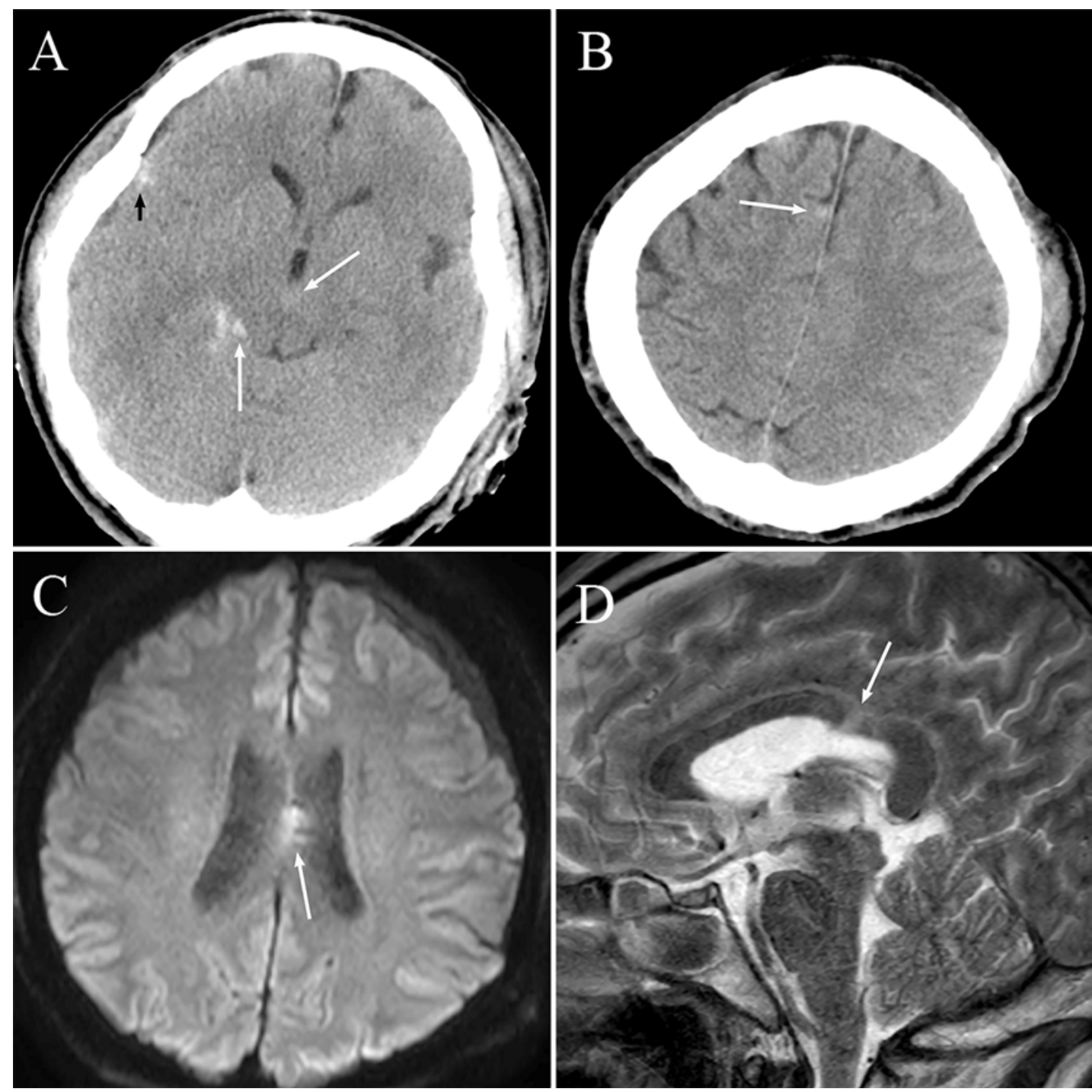

FIG. 1. Images obtained in a 41-year-old man who was a victim of a car accident. On admission to the emergency department, his GCS score was 7/15. The initial CT performed 25 minutes after admission shows tSAH in the perimesencephalic cistern (A, white arrows), in the interhemispheric fissure (B, arrow), and in the right sylvian fissure (A, black arrow) and vallecula (not shown) that led to a tSAH score of 4 . No hemorrhagic mass such as a contusion was noted in the brain parenchyma. Initial CT also showed multiple facial fractures and hemosinuses (not shown). On MRI performed 10 days later, nonhemorrhagic DAI lesions are seen in the corpus callosum on DWI (C, arrow) and on T2-weighted MRI (D, arrow; DAI stage 2 = severe DAI). The patient showed a poor clinical outcome at hospital discharge and 6 months later.

was associated with both overall DAI disease and severe DAI, and its absence had high specificity $(81.7 \%)$ and high NPV (89.9\%) in excluding severe DAI. In addition, we showed that midline tSAH was independently associated with poor early and long-term outcome.

Posttraumatic brain lesions occur mainly by two major mechanisms: contact with the skull (direct injuries) and shearing strain (indirect injuries). ${ }^{7}$ The association between IVH and DAI has been attributed to the fact that both are produced mainly by the latter mechanism. ${ }^{18,19}$ The association found in this study between midline tSAH and severe DAI suggests that some midline superficial vessels might also be torn during the shearing strain: the rigid structures (clivus or falx) at midline could serve as pivotal points for vessel twisting, resulting in midline tSAH concomitantly with IVH and DAI. Thus, we believe that midline tSAH and IVH should be considered a marker of strong shearing forces.

To our knowledge, this is the first study to clearly establish the relationship between midline tSAH and severe DAI. Conversely, when midline tSAH is not present on initial CT, strong shearing strain may not be the main mechanism of TBI and severe DAI is less likely to be noted on MRI. This is supported by our results showing a relatively high specificity and high NPV of midline tSAH for severe DAI. However, among 51 patients with severe DAI, $11(21.6 \%)$ did not show tSAH and 9 (17.6\%) showed tSAH only in locations other than midline. These findings allowed us to speculate that SAH, which is a dynamic lesion, can circulate or resolve, especially in patients scanned 

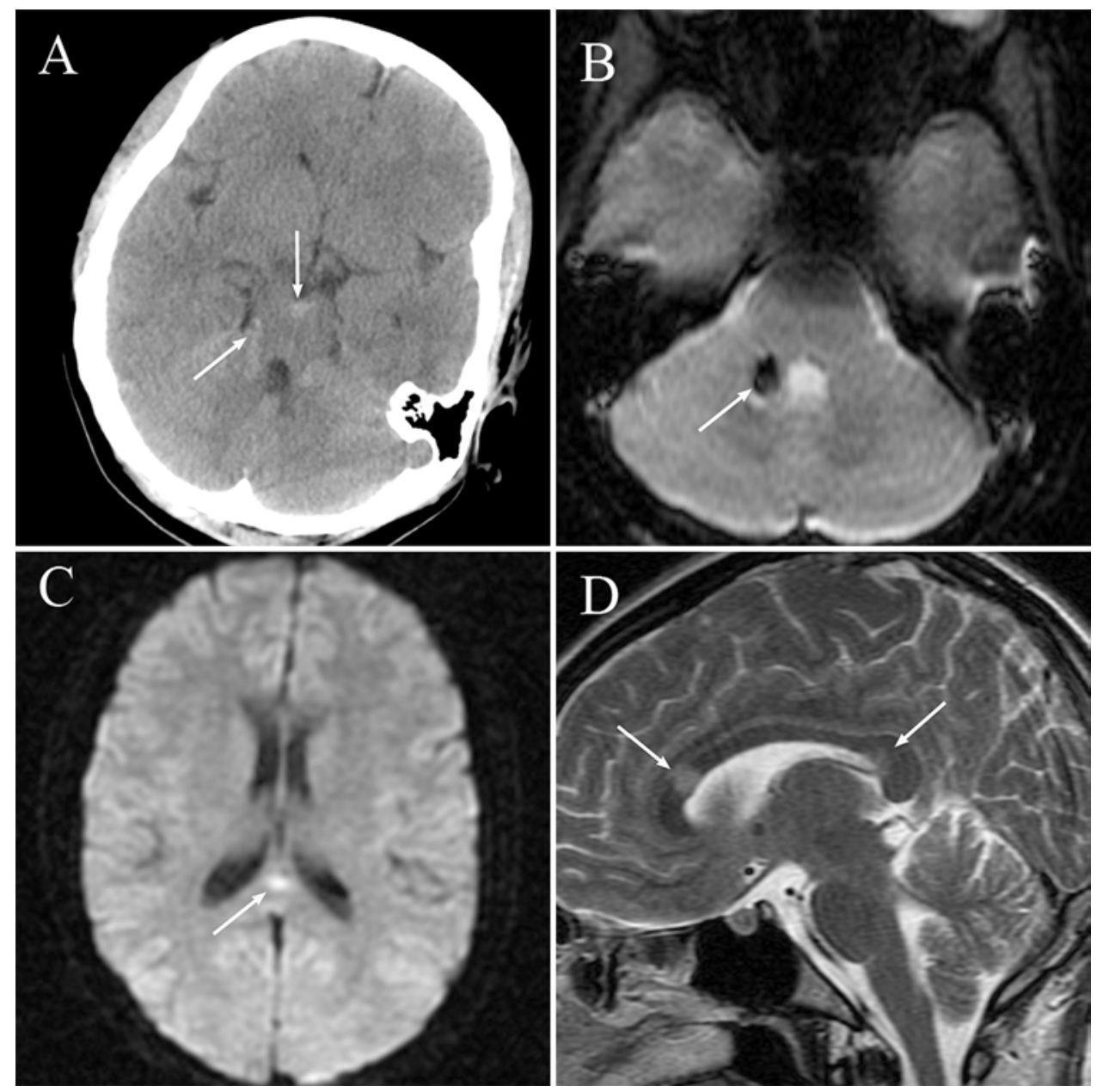

FIG. 2. Images from a 17-year-old woman who was a victim of a car accident. Her initial GCS score at admission to the emergency room was 6/15. The initial CT scan (A) performed 30 minutes after onset shows perimesencephalic SAH (arrows) as the only lesion (tSAH score = 1). MRI was subsequently performed 7 days later. Axial T2*-weighted MRI (B) shows hypointense foci (arrow) in the right lateral aspect of the brainstem, consistent with hemorrhagic DAI. Axial DWI (C) shows high signal intensity in the splenium (arrow). Sagittal T2-weighted MRI (D) revealed hyperintense foci in the corpus callosum (arrows). This patient had a midline $\mathrm{SAH}$ on initial CT, and the subsequent MRI revealed the diagnosis of DAI stage 3 (the most severe DAI). The patient showed a poor clinical outcome at hospital discharge and 6 months later.

late after onset of trauma. This could explain the relatively lower sensitivity and PVV of tSAH for severe DAI noted in this study. With this in mind, in the absence of midline SAH, clinical parameters (specifically GCS score) should also be considered to support the indication or not to undergo subsequent MRI.

We also believe that our results could explain an important clinical question that has persisted for a few decades: "Why do some patients with tSAH show poor clinical outcome?" 14,16 Our finding regarding the association between midline tSAH and both severe DAI and early or long-term poor GOSE score suggests that poor outcomes observed in some patients with tSAH are probably due not to tSAH itself but to severe DAI that is not readily detected by initial $\mathrm{CT}$, for which midline $\mathrm{SAH}$ is a probable surrogate.

In the literature there have been a few studies that have assessed the predictive value of initial CT findings with regard to DAI..$^{2,18,19}$ Two recent studies have shown that IVH, but not tSAH, was the only predictor of DAI on initial $\mathrm{CT},{ }^{18,19}$ whereas in our present study, both tSAH and IVH were independent predictors of DAI. The discrepancies of these results might be explained by differences in inclusion criteria, as the 2 other studies were based only on patients with a clinical diagnosis of TBI (excluding patients with facial injury), whereas the present study included patients with a reported history of trauma anywhere in the head region who received both brain CT and MRI. Our inclusion criterion was also guided by a previous study that has shown an association between facial fracture and DAI. ${ }^{27}$ Inclusion of patients with facial trauma increased the number of mild TBI cases (71.9\% in the present study vs $63.9 \%$ in 1 of the previous series ${ }^{18}$ ). Although tSAH can result from both direct and indirect injuries, tSAH is more likely to result after severe rather than more mild cases of TBI, which we suspect has blurred the statistical significance of tSAH from indirect injuries in previous studies. By including patients with facial trauma, the present study might have alleviated this blurring effect and therefore revealed the strong association between tSAH and DAI. From these conflicting results, it is possible that even facial trauma 
TABLE 4. Results of multivariate logistic regression where the 6 locations of $\mathrm{TSAH}$ on initial CT and 5 other initial CT findings (excluding the variable "tSAH") were used as candidate predictors of DAI on subsequent MRI

\begin{tabular}{|c|c|c|c|c|}
\hline \multirow[b]{2}{*}{ CT items } & \multirow{2}{*}{$\begin{array}{l}\text { No. } \\
(\%)\end{array}$} & \multicolumn{3}{|c|}{ Multivariate Analysis } \\
\hline & & Adjusted OR & $95 \% \mathrm{Cl}$ & $p$ Value \\
\hline Basal cistern status* & $4(1)$ & 1.7 & $0-235.4$ & 0.8219 \\
\hline Midline shift & $15(6)$ & 1.1 & $0-80.1$ & 0.9550 \\
\hline EDH & $19(7)$ & 0.7 & $0.2-2.5$ & 0.5589 \\
\hline Cortical tSAH & $100(37)$ & 1.6 & $0.6-4.1$ & 0.3217 \\
\hline Sylvian fissure tSAH & $69(26)$ & 1.6 & $0.6-4.1$ & 0.3191 \\
\hline Sylvian vallecula tSAH & $50(19)$ & 1.2 & $0.46-3.35$ & 0.6818 \\
\hline $\begin{array}{l}\text { Interhemispheric fis- } \\
\text { sure tSAH }\end{array}$ & $27(10)$ & 7.4 & $2-32.8$ & 0.0009 \\
\hline Cerebellar folia tSAH & $13(5)$ & 1.2 & $0.2-5.5$ & 0.8576 \\
\hline $\begin{array}{c}\text { Perimesencephalic } \\
\text { cisterns tSAH }\end{array}$ & $57(21)$ & 4.6 & $1.9-11.7$ & 0.0005 \\
\hline IVH & $28(10)$ & 4.1 & $1.4-12.6$ & 0.0083 \\
\hline $\begin{array}{l}\text { Volume of hemor- } \\
\text { rhagic mass }\end{array}$ & $14(5)$ & 0.6 & $0-3.8$ & 0.5575 \\
\hline
\end{tabular}

Boldface type indicates statistical significance.

* Values shown represent changes noted from level 0 to level 2 in the ordinal variable.

may lead to tSAH and/or DAI, but this is outside the scope of the present study. A dedicated study is necessary to better understand the relationship between the severity and/or location of facial trauma and DAI.

Our study has some limitations. First, most patients did not receive SWI in addition to T2*-weighted MRI, which might have caused an undercount of hemorrhagic DAI lesions. However, our protocol is consistent with almost all previous studies that assessed this "DAI grading," in that they used T2*-weighted MRI, not SWI, 10,18,20,23 thereby allowing comparison. Also, the term "DAI" in this study was used for not only hemorrhagic DAI but also nonhemorrhagic DAI, the latter of which represented more than $80 \%$ of overall DAI disease in this study, consistent with previous reports. ${ }^{5,9,21}$ Therefore, we believe that the lack of SWI in most of the patients may not significantly compromise the information regarding the "presence or not of DAI," which this study wishes to convey. Second, of the 1189 patients who underwent CT at admission, only 270 subsequently underwent MRI. This was due to milder injuries in the other patients with no clinical indication for further investigation, a finding that is consistent with the daily clinical practice in Japan. Third, our sample had a relatively small number of patients with DAI $(n=77)$, which could affect the statistical power of some of our multiple logistic regressions. ${ }^{22}$

\section{Conclusions}

Midline tSAH could implicate the same shearing mechanism that underlies severe DAI, for which midline tSAH is a probable surrogate. Therefore, the presence of midline tSAH on initial CT was associated with poor early and long-term outcomes, probably due to severe DAI. Con- versely, the absence of midline tSAH on initial CT was a reliable marker to exclude severe DAI.

\section{Acknowledgments}

We are grateful to Asaka Ishigamori, MA, Keiko Furuya, RN, Arata Nagai, and Tadahisa Shishido for their excellent technical support.

\section{References}

1. Adams JH, Doyle D, Ford I, Gennarelli TA, Graham DI, McLellan DR: Diffuse axonal injury in head injury: definition, diagnosis and grading. Histopathology 15:49-59, 1989

2. Beretta L, Anzalone N, Dell'Acqua A, Calvi MR, Gemma M: Post-traumatic interpeduncular cistern hemorrhage as a marker for brainstem lesions. J Neurotrauma 27:509-514, 2010

3. Calvi MR, Beretta L, Dell'Acqua A, Anzalone N, Licini G, Gemma M: Early prognosis after severe traumatic brain injury with minor or absent computed tomography scan lesions. J Trauma 70:447-451, 2011 (Erratum in J Trauma 70:1582, 2011)

4. Gean AD: Maxillofacial trauma, in Imaging of Head Trauma. New York: Raven Press, 1995, pp 427-493

5. Gentry LR, Godersky JC, Thompson B: MR imaging of head trauma: review of the distribution and radiopathologic features of traumatic lesions. AJR Am J Roentgenol 150:663672,1988

6. Hilario A, Ramos A, Millan JM, Salvador E, Gomez PA, Cicuendez M, et al: Severe traumatic head injury: prognostic value of brain stem injuries detected at MRI. AJNR Am J Neuroradiol 33:1925-1931, 2012

7. Holbourn AHS: Mechanics of head injuries. Lancet 2:438 441,1943

8. Jayakumar PN, Kolluri VR, Basavakumar DG, Arya BY, Das BS: Prognosis in traumatic intraventricular haemorrhage. Acta Neurochir (Wien) 106:48-51, 1990

9. Kim JJ, Gean AD: Imaging for the diagnosis and management of traumatic brain injury. Neurotherapeutics 8:39-53, 2011

10. Lagares A, Ramos A, Pérez-Nuñez A, Ballenilla F, Alday R, Gómez PA, et al: The role of MR imaging in assessing prognosis after severe and moderate head injury. Acta Neurochir (Wien) 151:341-356, 2009

11. Lee JP, Lui TN, Chang CN: Acute post-traumatic intraventricular hemorrhage analysis of 25 patients with emphasis on final outcome. Acta Neurol Scand 84:85-90, 1991

12. Lin TK, Tsai HC, Hsieh TC: The impact of traumatic subarachnoid hemorrhage on outcome: a study with grouping of traumatic subarachnoid hemorrhage and transcranial Doppler sonography. J Trauma Acute Care Surg 73:131-136, 2012

13. Lingsma HF, Roozenbeek B, Steyerberg EW, Murray GD, Maas AI: Early prognosis in traumatic brain injury: from prophecies to predictions. Lancet Neurol 9:543-554, 2010

14. Maas AI, Hukkelhoven CW, Marshall LF, Steyerberg EW: Prediction of outcome in traumatic brain injury with computed tomographic characteristics: a comparison between the computed tomographic classification and combinations of computed tomographic predictors. Neurosurgery 57:11731182,2005

15. Maas AI, Stocchetti N, Bullock R: Moderate and severe traumatic brain injury in adults. Lancet Neurol 7:728-741, 2008

16. Marshall LF, Marshall SB, Klauber MR, Clark MV, Eisenberg HM, Jane JA, et al: A new classification of head-injury based on computerized-tomography. J Neurosurg 75 (1 Suppl):S14-S20, 1991

17. Mata-Mbemba D, Mugikura S, Nakagawa A, Murata T, Ishii $\mathrm{K}, \mathrm{Li} \mathrm{L}$, et al: Early CT findings to predict early death in 
patients with traumatic brain injury: Marshall and Rotterdam CT scoring systems compared in the major academic tertiary care hospital in northeastern Japan. Acad Radiol 21:605611, 2014

18. Mata-Mbemba D, Mugikura S, Nakagawa A, Murata T, Kato Y, Tatewaki Y, et al: Intraventricular hemorrhage on initial computed tomography as marker of diffuse axonal injury after traumatic brain injury. J Neurotrauma 32:359-365, 2015

19. Matsukawa H, Shinoda M, Fujii M, Takahashi O, Murakata A, Yamamoto D, et al: Intraventricular hemorrhage on computed tomography and corpus callosum injury on magnetic resonance imaging in patients with isolated blunt traumatic brain injury. J Neurosurg 117:334-339, 2012

20. Matsukawa H, Shinoda M, Fujii M, Takahashi O, Yamamoto D, Murakata A, et al: Genu of corpus callosum in diffuse axonal injury induces a worse 1-year outcome in patients with traumatic brain injury. Acta Neurochir (Wien) 153:1687-1694, 2011

21. Meythaler JM, Peduzzi JD, Eleftheriou E, Novack TA: Current concepts: diffuse axonal injury-associated traumatic brain injury. Arch Phys Med Rehabil 82:1461-1471, 2001

22. Novikov I, Fund N, Freedman LS: A modified approach to estimating sample size for simple logistic regression with one continuous covariate. Stat Med 29:97-107, 2010

23. Skandsen T, Kvistad KA, Solheim O, Strand IH, Folvik M, Vik A: Prevalence and impact of diffuse axonal injury in patients with moderate and severe head injury: a cohort study of early magnetic resonance imaging findings and 1-year outcome. J Neurosurg 113:556-563, 2010

24. Smith DH, Meaney DF, Shull WH: Diffuse axonal injury in head trauma. J Head Trauma Rehabil 18:307-316, 2003

25. Tong KA, Ashwal S, Holshouser BA, Shutter LA, Herigault G, Haacke EM, et al: Hemorrhagic shearing lesions in children and adolescents with posttraumatic diffuse axonal injury: improved detection and initial results. Radiology 227:332-339, 2003

26. Wilson JT, Pettigrew LE, Teasdale GM: Structured interviews for the Glasgow Outcome Scale and the extended Glasgow Outcome Scale: guidelines for their use. J Neurotrauma 15:573-585, 1998

27. Zwahlen RA, Labler L, Trentz O, Grätz KW, Bachmann LM: Lateral impact in closed head injury: a substantially increased risk for diffuse axonal injury-a preliminary study. J Craniomaxillofac Surg 35:142-146, 2007

\section{Disclosures}

The authors report no conflict of interest concerning the materials or methods used in this study or the findings specified in this paper.

\section{Author Contributions}

Conception and design: Mugikura, Tominaga. Acquisition of data: Mugikura, Mata-Mbemba, Nakagawa, Murata. Analysis and interpretation of data: Mugikura, Mata-Mbemba, Nakagawa, Murata, Kushimoto, Takahashi, Takase. Drafting the article: Mugikura, Mata-Mbemba, Takahashi. Critically revising the article: Mugikura, Mata-Mbemba, Nakagawa, Ishii, Kushimoto, Tominaga, Takase. Reviewed submitted version of manuscript: all authors. Approved the final version of the manuscript on behalf of all authors: Mugikura. Study supervision: Nakagawa, Tominaga.

\section{Correspondence}

Shunji Mugikura: Tohoku University Graduate School of Medicine, Sendai, Japan. email: mugi@rad.med.tohoku.ac.jp. 